Journal of Advanced Research in Applied Sciences and Engineering Technology

\title{
Radiative Boundary Layer Flow of Casson Fluid Over an Exponentially Permeable Slippery Riga Plate with Viscous Dissipation
}

\author{
Nur Syamila Yusof ${ }^{1}$, Siti Khuzaimah Soid ${ }^{1,}{ }^{*}$, Mohd Rijal Illias ${ }^{1}$, Ahmad Sukri Abd Aziz ${ }^{1}$, Nor Ain \\ Azeany Mohd Nasir ${ }^{2}$ \\ Faculty of Computer and Mathematical Scieces, Universiti Teknologi MARA, 40450 Shah Alam, Selangor, Malaysia \\ Department of Mathematics, Center for Defence Foundation Studies, National Defence University of Malaysia, Kem Sungai Besi, 57000 Kuala \\ Lumpur, Malaysia
}

\section{ARTICLE INFO}

Article history:

Received 29 June 2020

Received in revised form 10 August 2020

Accepted 14 September 2017

Available online 17 November 2020

Keywords:

Casson fluid model; boundary layer flows; permeable slippery Riga plate; radiative heat transfer; stretching/shrinking sheet;

\section{ABSTRACT}

This study is aimed to analyze the steady of stagnation point flow and radiative heat transfer of a non-Newtonian fluid which is Casson fluid passing over an exponentially permeable slippery Riga plate in presence of thermal radiation, magnetic field, velocity slip, thermal slip, and viscous dissipation effects. The governing partial differential equations are transformed into ordinary differential equations by using similarity transformation then solved numerically by boundary value problem solver (BVP4C) in MATLAB software package. The numerical results are evaluated with previous researches to reach an agreement with the parameters of the current study. This study is discussing the behavior of the velocity and temperature profiles as well as skin friction coefficient and local Nusselt number for various physical parameters such as magnetic field, radiation, suction, thermal slip, velocity slip, Prandtl number, Eckert number and modified Hartmann number. Numerical results are shown graphically for each parameter with different values. It is found that the momentum boundary layer thickness increases with increasing the values of Casson parameter. The temperature decreases when the velocity slip parameter and thermal slip parameter are increased.

\section{Introduction}

In this world, there is almost everything covered with fluids even the living of nature. Water is well-known as one kind of fluid. The liquid is one of the substances with zero shear force when there is no force presence to the fluid flow along to the surface. Newtonian fluids are the fluids that obey the Newtonian law of viscosity. However, there are some fluids that are invalid to describe the

\footnotetext{
* Corresponding author.

E-mail address: khuzaimah@tmsk.uitm.edu.my (Siti Khuzaimah Soid)
} 
rheological behavior of several important materials in the industrial sector such as ketchup, blood clotting, several oils, concentrated fruit juices, honey, and many other examples. Those invalid applications in the theory of Newtonian fluids are known as non-Newtonian fluid. Recently, the study of non-Newtonian fluids has become attention where it has a variety of applications in industrial and engineering sectors especially in food processing.

One of the non-Newtonian fluids is Casson fluid. Casson fluid was introduced by Casson [1] to stimulate industrial inks, thus this model has significant applications in polymers processing industries and biomechanics. The heat transfer and stagnation point flow over a stretching/shrinking sheet are the crucial investigation within the researchers due to its wide range of applications in the industrial and engineering sectors. Some of these applications are paper production, glass blowing, cooling of metallic sheets, jet emerging from slot-jets, and flow over the tips of the submarine. An exact analytical solution of the boundary layer past a stretching sheet was first introduced by Crane [2] and the boundary layer flow over a shrinking sheet was evolved by Wang [3]. The fluid flow and heat transfer are influenced by the value of the Casson parameter in the presence of suction/injection at the surface were analyzed by Pramanik [4] through a horizontal exponentially stretching sheet in the presence of porous medium and thermal radiation, meanwhile the inclined linear stretching sheet in nanofluid was studied by Rawi et al., [5].

In recent years, the nonlinear boundary layer has been subjected to the researchers to conduct the problems related to the fluid flow whereas it is important to discover since there is a wide range in the applications based on the boundary layer theory. Slip velocity exists when there is a velocity gradient occurs on the surface in which the fluid flow moves freely on the surface such as gels, emulsions, and foams [6]. Furthermore, the thermal radiation effects on heat transfer over a stretching/shrinking surface is an essential role in engineering since there is a wide range in applications such as liquid metal fluids, gas turbines and aircraft. Srinivas et al., [7] conducted the effect of thermal radiation and chemical reaction on the unsteady flow of a micropolar fluid over a porous stretching sheet. Another study about the effects of velocity slip and radiation on the stagnation point flow and heat transfer past a permeable stretching/shrinking Riga plate has been carried out by Nasir et al., [8].

Viscous dissipation defined by Hussanan et al., [9] is a process in which the work done by a fluid on adjacent layers due to the action of shear forces. Soid et al., [10] carried out the study of an unsteady magneto-hydrodynamic flow and heat transfer over a shrinking sheet with ohmic heating. The study was extended the work from Bhattacharyya [11] where the effect of magnetic field, viscous dissipation and ohmic heating are calculating on the problem. The nonlinear viscous dissipation is an important topic to discuss because it is responsible for the instability during the heat transfer process. In other words, viscous dissipation is an internal heat generation mechanism where it can lead to unstable temperature distribution [12]. Moreover, there is a combination of permanent magnets and a spanwise aligned array of alternating electrodes mounted on a plane surface which called as a Riga plate.

Riga magnet is used as an efficient agent to minimize the skin friction and pressure drag of submarines. The fluid flows under the influence of magnetic force along the Riga plate have huge applications in engineering and biomechanics. To illustrate the characteristics of laminar fluid flow due to Riga plate have been carried out in various physical aspects such as Islam et al., [13] investigated the behavior of fluid flow with the presence of magnetic force in a rotating system on a Riga plate. Another study on the convective Casson fluid flow past a Riga plate was conducted by Loganathan and Deepa [14]. The research was focused on the behavior of non-linear flow under radiation, permeability and the surface parallel to Lorentz force. A study on the heat transfer in 
Casson fluid over an exponentially permeable shrinking sheet by Haldar et al., [15] was found that wall temperature declines with the increase values of convective parameter.

The boundary layer flow with slip condition at the surface has become huge attention nowadays and has been analyzed under various physical conditions [16-19]. However, there are several uncertain behaviors in the manufacturing process that could not be avoided or controlled especially during the process of trespassing flow at a critical point. Therefore, the study of nonlinear stagnation point flow and heat transfer are taken into this problem to give the approximated solutions based on real problems. In this paper, we extend the work of Haldar et al., [15] to examine the effects of Riga magnetic field, viscous dissipation, stagnation point flow, thermal radiation, velocity and thermal slip boundary on the problem of steady heat transfer of Casson fluid over an exponentially stretching/shrinking permeable sheet. This study concerns on the realistic applications and pointed out that the theory of Casson fluid is essential to introduce and describe the non-Newtonian behaviour of certain fluids.

The study on the heat transfer over a permeable stretching/shrinking Riga plate in the Casson fluid with the presence of viscous dissipation, radiation and slip effects parameter will enhance the understanding of the behavior of these flows. The governing partial differential equations are reduced into a system of nonlinear ordinary differential equations by applying similarity transformations. Numerical results are obtained by using BVP4C solver in the MATLAB software for different values for each governing parameter. The skin friction coefficient and local Nusselt number are analyzed and shown in tabular forms and graphs.

\section{Mathematical Formulation}

Consider a steady two-dimensional stagnation point flow and heat transfer of Casson fluid over a stretching/shrinking Riga plate. The physical model in Figure 1 is shown in Cartesian coordinates where $x$ and $y$ are measured over a Riga plate and normal to it respectively. The velocity of the stretching/shrinking surface is $U_{w}(x)=U_{0} e^{\frac{x}{L}}$ where $U_{0}$ is the reference velocity while the straining velocity $U_{e}(x)$ is representing the velocity of the flow far from the sheet (inviscid flow) and $L$ is a characteristic length. The temperature of the sheet is $T_{w}=T_{\infty}+T_{0} e^{\frac{x}{2 L}}$, where the constant temperature of the ambient fluid is $T_{\infty}$ and $T_{0}$ is the reference temperature. $M=M_{0} e^{\frac{2 x}{L}}$ is a variable of magnetic field where $M_{0}$ is a constant. Assuming the rheological equation of state for an isotropic and incompressible flow of a Casson fluid is as follow:

$\tau_{i j}= \begin{cases}2\left(\mu_{B}+p_{y} / \sqrt{2 \pi}\right) e_{i j,} & \pi>\pi_{c} \\ 2\left(\mu_{B}+p_{y} / \sqrt{2 \pi_{c}}\right) e_{i j,} & \pi>\pi_{c},\end{cases}$

where $\mu_{B}$ is the plastic dynamic viscosity of the non-Newtonian fluid, $p_{y}$ is the yield stress of the fluid, $\pi=e_{i j} e_{i j}, e_{i j}$ is the $(i, j)$ th component of the deformation rate with itself and $\pi_{c}$ denotes a critical value of this product based on the non-Newtonian model [20-21]. 


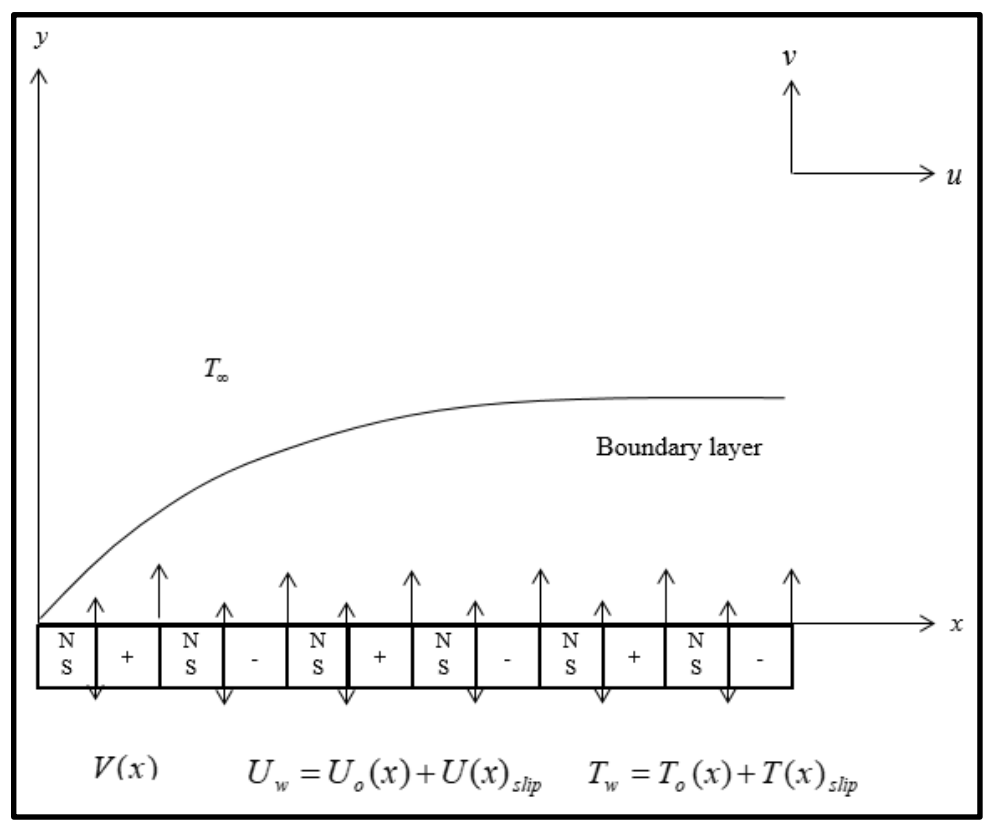

Fig. 1. Physical model of the boundary layer on the Riga plate

The governing equations of the steady stagnation boundary layer equations for the continuity and momentum of this problem can be written as below:

$\frac{\partial u}{\partial x}+\frac{\partial v}{\partial y}=0$

$u \frac{\partial u}{\partial x}+v \frac{\partial u}{\partial y}=u_{e} \frac{\partial u_{e}}{\partial x}+v\left(1+\frac{1}{\beta}\right) \frac{\partial^{2} u}{\partial y^{2}}+\frac{\pi j_{0} M}{8 \rho} e^{\left(-\frac{\pi}{\alpha_{1}} y\right)}$

$u \frac{\partial T}{\partial x}+v \frac{\partial T}{\partial y}=\alpha \frac{\partial^{2} T}{\partial y^{2}}+\frac{1}{\rho C_{p}} \frac{\partial q_{r}}{\partial y}+\frac{\mu}{\rho C_{p}}\left(1+\frac{1}{\beta}\right)\left(\frac{\partial u}{\partial y}\right)^{2}$

with the boundary conditions

$u=u_{w}(x)=\lambda U_{w}(x)+N \frac{\partial u}{\partial y}, \quad v=-V_{w}(x), T=T_{w}+D \frac{\partial T}{\partial y}, \quad$ at $y=0$

$u \rightarrow U_{e}(x)=U_{0} e^{\frac{x}{L}}, \quad T \rightarrow T_{\infty}$, as $y \rightarrow \infty$.

where $u$ and $v$ are component of velocity in $x$-axis and $y$-axis respectively, $v$ is the kinematic viscosity, $\beta$ is the Casson fluid, $\rho$ is fluid density, $j_{0}$ is the applied current density in the electrodes, $\alpha_{1}$ is the width for electrodes and magnets, $\alpha=\frac{k}{\rho C_{p}}$ is the thermal diffusivity where $k$ is the thermal conductivity, $C_{p}$ is the specific heat at constant pressure and $\lambda$ is the constant stretching/shrinking parameter where $\lambda>0$ refers to stretching while $\lambda<0$ is for shrinking sheet. It is assumed that $N=N_{1} e^{-\frac{x}{2 L}}$ is the velocity slip factor changes with $x$ while $N_{1}$ is the initial value of velocity slip and $D=D_{1} e^{-\frac{x}{2 L}}$ is the thermal slip factor changes with $x$ and $D_{1}$ is the initial value of the thermal slip factor. 
$V_{w}(x)=V_{0} e^{\frac{x}{2 L}}$ is the velocity at the surface where $V_{0}$ is the initial strength when $V_{0}>0$ for injection while $V_{0}<0$ for suction and $T$ is the temperature parameter.

The radiative heat flux $q_{r}$ under Rosseland approximation for thermal radiation [22] is then simplified as

$q_{r}=-\frac{4 \sigma^{*}}{3 k^{*}} \frac{\partial T^{4}}{\partial y}$

where, $\sigma^{*}$ is the Stefan-Boltzman constant and $k^{*}$ is the absorption coefficient. The temperature differences within the flow such as the term $T^{4}$ are expressed as linear function of temperature. Hence, expanding $T^{4}$ in a Taylor series about $T_{\infty}$ and neglecting higher-order terms will be as follows.

$T^{4} \cong 4 T_{\infty}^{3} T-3 T_{\infty}^{4}$.

Using Eqs. (5) and (6), Eq. (3) can be written as

$u \frac{\partial T}{\partial x}+v \frac{\partial T}{\partial y}=\alpha \frac{\partial^{2} T}{\partial y^{2}}+\frac{16 \sigma^{*} T_{\infty}^{3}}{3 k^{*} \rho C_{p}} \frac{\partial^{2} T}{\partial y^{2}}+\frac{\mu}{\rho C_{p}}\left(1+\frac{1}{\beta}\right)\left(\frac{\partial u}{\partial y}\right)^{2}$.

We assume the following similarity variables $\eta$, the velocity components $u, v$, and the dimensionless temperature $\theta$ can be considered for Eqs. (1), (2) and (7):

$$
\begin{aligned}
& \psi=\sqrt{2 U_{0} \nu L} e^{\frac{x}{2 L}} f(\eta), \quad \theta(\eta)=\frac{T-T_{\infty}}{T_{W}-T_{\infty}}, \quad \eta=\sqrt{\frac{U_{0}}{2 v L}} e^{\frac{x}{2 L} y} \\
& u=U_{O} e^{\frac{x}{L}} f^{\prime}(\eta), \quad v=-\sqrt{\frac{v U_{0}}{2 L}} e^{\frac{x}{2 L}}\left(f(\eta)+\eta f^{\prime}(\eta)\right) .
\end{aligned}
$$

Substituting the similarity variables from Eq. (8) into Eqs. (1), (2) and (7) are reduced into the following ordinary differential equations as below:

$$
\begin{aligned}
& \left(1+\frac{1}{\beta}\right) f^{\prime \prime \prime}(\eta)+2-2 f^{\prime 2}(\eta)+f(\eta) f^{\prime \prime}(\eta)+Q e^{(-A \eta)}=0, \\
& \left(1+\frac{4}{3} R d\right) \theta^{\prime \prime}(\eta)+\operatorname{Pr} \theta^{\prime}(\eta) f(\eta)-\operatorname{Pr} f^{\prime}(\eta) \theta(\eta)+\operatorname{Pr} E c\left(1+\frac{1}{\beta}\right) f^{\prime \prime 2}(\eta)=0
\end{aligned}
$$

with the boundary condition

$$
\begin{aligned}
& f(0)=S, \quad f^{\prime}(0)=\lambda+\omega f^{\prime \prime}(0), \quad \theta(0)=1+\varepsilon \theta^{\prime}(0), \text { at } \eta=0 \\
& f^{\prime}(\infty) \rightarrow 1, \quad \theta(\infty) \rightarrow 0, \text { as } y \rightarrow \infty
\end{aligned}
$$

where primes denote differentiation with respect to $\eta, \beta$ is the Casson fluid parameter, $Q$ is the modified Hartmann number, $A$ is the dimensionless parameter, $R d$ is the radiation parameter, $\operatorname{Pr}$ is the Prandtl number, $E c$ is the Eckert number, $S$ is the suction/injection parameter, $\lambda$ is the 
stretching/shrinking parameter, $\omega$ is the velocity slip factor and $\varepsilon$ is the thermal slip factor, which are defined as

$$
\begin{aligned}
& \operatorname{Pr}=\frac{k}{\mu C_{p}}, \quad R d=\frac{4 \sigma^{*} T_{\infty}^{3}}{k^{*} k}, \quad E c=\frac{U_{0}^{2}}{T_{0} C_{p}} e^{\frac{3 x}{2 L}}, \quad Q=\frac{\pi j_{0} M_{0}}{8 \rho U_{0}^{2}}, \quad A=\frac{\pi}{\alpha_{1}} \cdot \frac{1}{e^{\frac{x}{2 L}}} \sqrt{\frac{2 v L}{U_{0}}}, \\
& M=M_{0} e^{\frac{2 x}{L}}, \quad S=v_{0} \sqrt{\frac{2 L}{v U_{0}}}, \quad \omega=N_{1} \sqrt{\frac{U_{0}}{2 v L}}, \quad \varepsilon=D_{1} \sqrt{\frac{U_{0}}{2 v L}} .
\end{aligned}
$$

The quantities of physical interest in this problem are the skin friction coefficient $C_{f}$ and the local Nusselt number $\mathrm{Nu}$ which are defined as

$$
C_{f}=\frac{\tau_{w}}{\rho U_{w}^{2}(x)}, \quad N u=\frac{x q_{w}}{k\left(T_{w}-T_{\infty}\right)}
$$

where $\tau_{w}$ is the shear stress or skin friction and $q_{w}$ is the heat flux from the surface of the sheet which are defined as

$\tau_{w}=\left(\mu_{B}+\frac{p_{y}}{\sqrt{2 \pi_{c}}}\right)\left(\frac{\partial u}{\partial y}\right)_{y=0}, \quad q_{w}=-k\left(1+\frac{4}{3} R d\right)\left(\frac{\partial T}{\partial y}\right)_{y=0}$.

Substituting Eqs. (8) and (14) into Eq. (13) and obtain:

$$
\sqrt{\frac{2 L}{x}} \frac{\sqrt{\operatorname{Re}}}{\left(1+\frac{1}{\beta}\right)} C_{f}=f^{\prime \prime}(0), \quad \frac{N u}{\sqrt{\frac{x}{2 L}} \sqrt{\operatorname{Re}}\left(1+\frac{4}{3} R d\right)}=-\theta^{\prime}(0)
$$

where $\operatorname{Re}=\frac{x U_{w}(x)}{v}$ is the local Reynolds number.

\section{Results and Discussion}

The nonlinear ordinary differential equations along with the boundary conditions were solved numerically using the BVP4C solver in MATLAB. The dimensionless parameters have been computed to perform numerical solutions which are Casson parameter $\beta$, Prandtl number $\operatorname{Pr}$, radiation parameter $R d$, Eckert number $E c$, modified Hartmann number $Q$, stretching/shrinking parameter $\lambda$, suction/injection parameter $S$, velocity slip factor $\omega$ and thermal slip factor $\varepsilon$ while the dimensionless parameter $A$ remains constant. The velocity and temperature profiles are determined graphically for a few parameters listed above as shown in Figures 2-9 meanwhile numerical results of skin friction coefficient and local Nusselt number are tabulated in Table 2.

In order to achieve the accuracy of the governing equations, the present study is being compared for the heat transfer coefficient with the numerical results of Haldar et al., [15] to validate the numerical results obtained for a steady case when $\operatorname{Pr}=0.7$, suction parameter remains constant at $S=5.0$ and Casson parameter $\beta$ is calculated with various values which are 1.0, 1.4, 2.0 and 2.4. The comparison in Table 1 shows that the heat transfer coefficient has achieved an agreement and prove 
that this research programming solver is strongly accurate and reliable in solving the nonlinear ordinary differential equations.

Table 1

Comparison of heat transfer coefficient, $-\theta^{\prime}(0)$ for the various value of $\beta$ and $\varepsilon$ as $Q=R d$ $=E c=A=\omega=0, S=5.0$ and $\operatorname{Pr}=0.7$ with $\lambda=-1$

\begin{tabular}{llll}
\hline & & Haldar et al., [15] & Present study \\
\cline { 3 - 4 }$\varepsilon$ & $\beta$ & $-\theta^{\prime}(0)$ & $-\theta^{\prime}(0)$ \\
\hline 10 & 1.0 & 0.097121 & 0.097121 \\
& 1.4 & 0.097129 & 0.097129 \\
& 2.0 & 0.097135 & 0.097135 \\
& 2.4 & 0.097138 & 0.097138 \\
\hline
\end{tabular}

The numerical results were evaluated by varying different values of several parameters while the dimensionless parameter, $A$ and other parameters is remaining constant at 1 . The shrinking parameter is consistently considered as $\lambda=-1$ while for the stretching parameter is $\lambda=1$ The results have been analyzed to discover the behavior of the skin friction coefficient, local Nusselt number, velocity profiles, and temperature profiles regarding various of physical parameters in this study. In this research, we have been decided the relative tolerance was set as $10^{-10}$ to obtain accurate numerical results and chose $\eta \rightarrow \infty$ as $\eta=4$ for the solution because it found suitable for the profiles to fulfil the boundary conditions asymptotically.

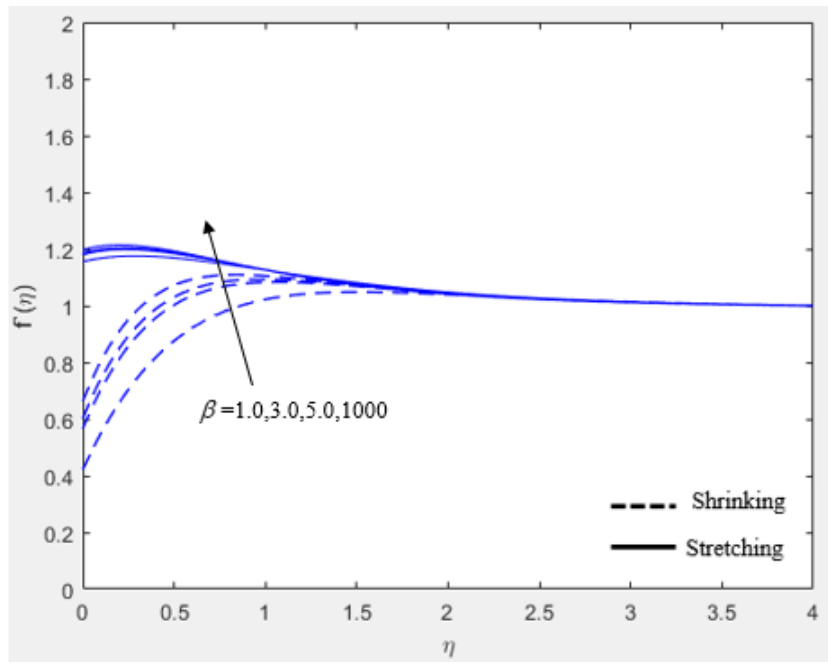

Fig. 2. Velocity profile, $f^{\prime}(\eta)$ for various values of $\beta$ when $Q=R d=E c=A=\omega={ }_{\varepsilon}=S=\operatorname{Pr}=1$

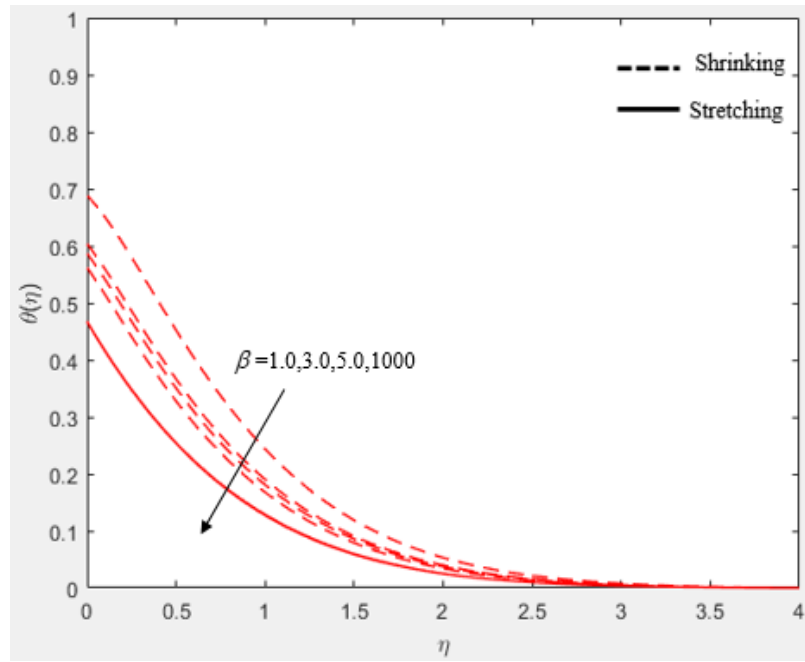

Fig. 3. Temperature profile, $\theta(\eta)$ for various values of $\beta$ when $Q=R d=E c=A=\omega={ }_{\varepsilon}=S=\operatorname{Pr}=1$

The effects of Casson parameter $\beta$ on the velocity and temperature profiles are depicted in Figures 2 and 3. It is analysed that the fluid velocity rises as the Casson fluid parameter increases initially and it continuous when the fluid moves along the shrinking sheet. Meanwhile, for stretching sheet, the movement of the fluid decreases after several time, but ultimately the velocity profiles towards 1 for both sheets. In fact, the high viscosity of the Casson fluid will lead to slow the fluid movement in the boundary layer due to the high drag force especially on the shrinking sheet. A consistent behaviour can be predicted for the Newtonian fluid as the Casson parameter approaches infinity. Opposite behaviour in Figure 3 which the temperature decreases as the values of the Casson parameter increases and it is obvious on the shrinking sheet. This is happening due to the viscosity 
of non-Newtonian fluid assists to decrease the temperature of the fluid in the boundary layer. Consequently, it causes to decrease the thermal boundary layer that represents the faster cooling.

Figures 4 and 5 show the velocity and temperature profiles for the Eckert number for both stretching and shrinking. The velocity distribution is constant because it is independent toward the momentum equation. However, it is significantly affecting the temperature distribution as depicted in Figure 5. The temperature distribution increases as the values of the Eckert number increase for both the stretching and shrinking surface. This is happening because the relationship between kinetic energy flow and the enthalpy can enhance the temperature of the boundary layer flow.

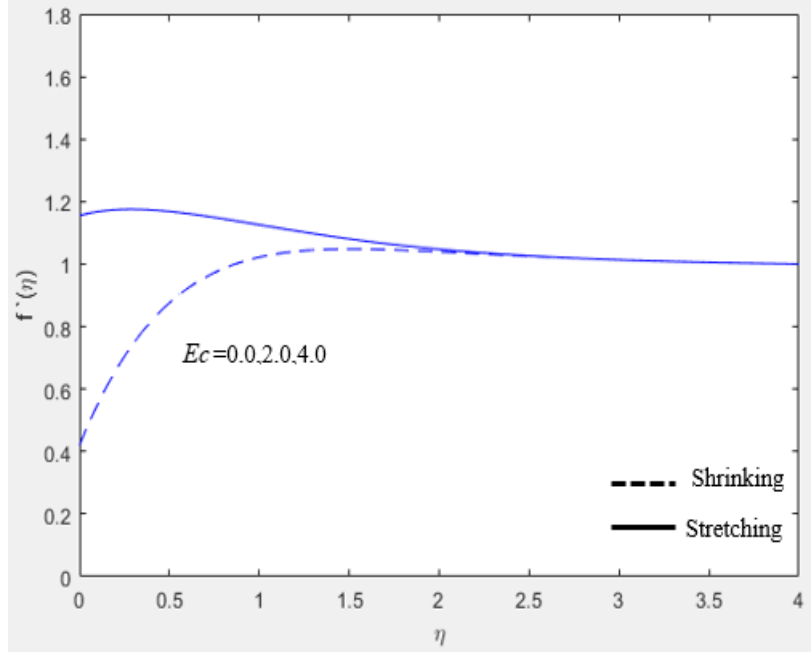

Fig. 4. Velocity profile, $f^{\prime}(\eta)$ for various values of Ec when $Q=R d=\beta=A=\omega=\varepsilon=S=\operatorname{Pr}=1$

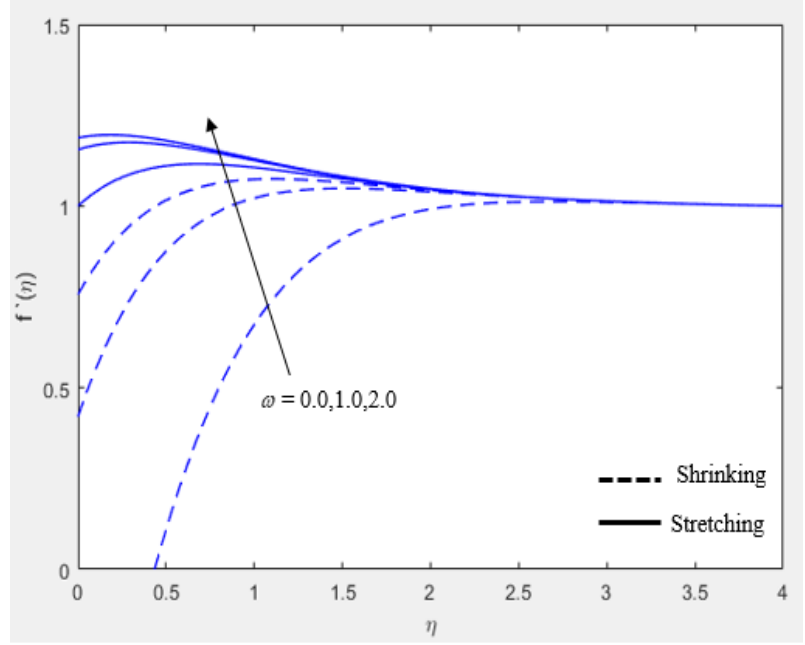

Fig. 6. Velocity profile, $f^{\prime}(\eta)$ for various values of $\omega$ when $Q=R d=\beta=A=E c=\varepsilon=S=\operatorname{Pr}=1$

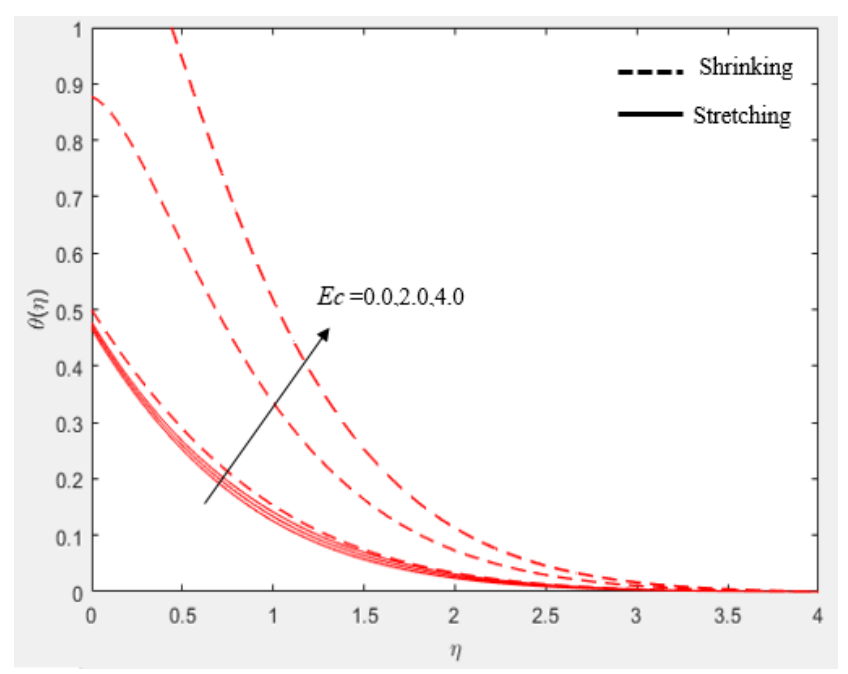

Fig. 5. Temperature profile, $\theta(\eta)$ for various values of $E c$ when $Q=R d=\beta=A=\omega=\varepsilon=S=\operatorname{Pr}=1$

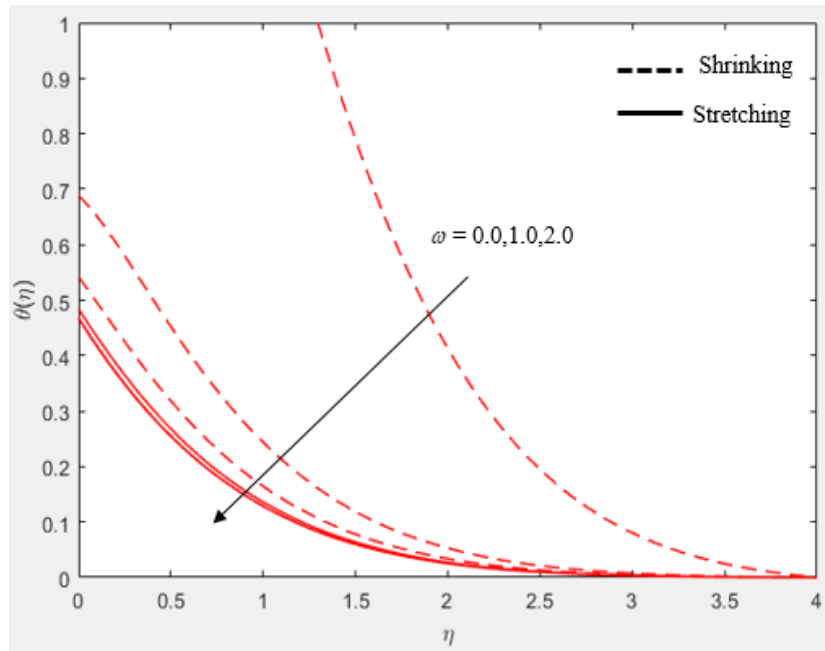

Fig. 7. Temperature profile, $\theta(\eta)$ for various values of $\omega$ when $Q=R d=\beta=A=E c={ }_{\varepsilon}=S=\operatorname{Pr}=1$

The effects of three different values of velocity slip parameter $\omega$ on the velocity and temperature profiles are defined in Figure 6 and Figure 7 respectively. Figure 6 represents the growth of the velocity slip parameter which will increase the fluid velocity within the boundary layer. It means that, the more slippery the surface is, the smoother the fluid flow. The temperature distributions reveal an opposite scenario which the temperature decreases as shown in Figure 7 . The decrease in the 
temperature is due to the higher viscosity of the fluid that resists the temperature to disperse. However, both figures result in reducing the velocity and thermal boundary layers.

Figure 8 states that the increase of the thermal slip parameter does not influence the fluid behavior in the boundary layer. However, it does affect the temperature profile as in Figure 9. Therefore, the thermal slip tends to lower the temperature distribution and simultaneously decrease the temperature boundary layer thickness. Near the boundary, the temperature distribution $\theta(0)$ is lower for the stretching sheet compared to shrinking sheet for a little increment in thermal slip. Surprisingly, $\theta(0)$ has a similar value for the higher slippery sheet.

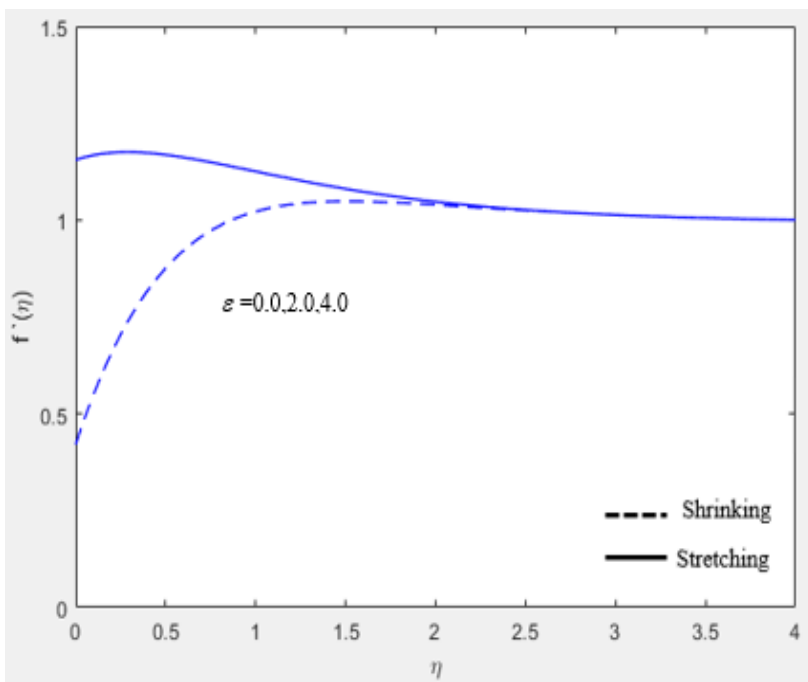

Fig. 8. Velocity profile, $f^{\prime}(\eta)$ for various values of $\varepsilon$ when $Q=R d=\beta=A=E c=\omega=S=\operatorname{Pr}=1$

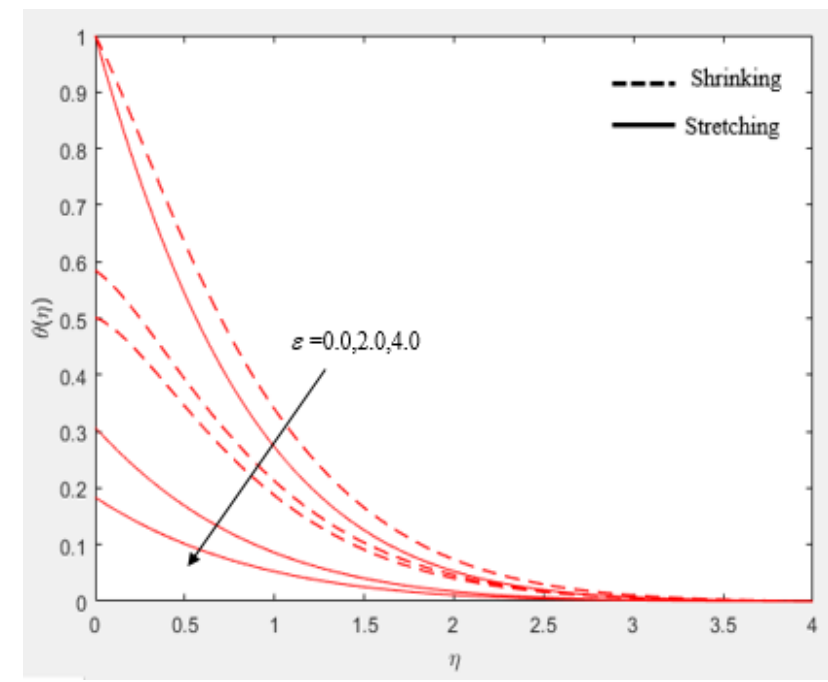

Fig. 9. Temperature profile, $\theta(\eta)$ for various values of $\varepsilon$ when $Q=R d=\beta=A=E c=\omega=S=\operatorname{Pr}=1$

\section{Table 2}

The values of skin friction coefficient, $f^{\prime \prime}(0)$ and local Nusselt number, $-\theta^{\prime}(0)$ for several values of $\beta$, $E c, \omega$ and $\varepsilon$ when $Q=R d=S=\operatorname{Pr}=A=1$

\begin{tabular}{llllllll}
\hline \multirow{2}{*}{$\beta$} & $E c$ & $\omega$ & $\varepsilon$ & \multicolumn{2}{l}{ stretching sheet $(\lambda>0)$} & \multicolumn{2}{l}{ shrinking sheet $(\lambda<0)$} \\
\cline { 5 - 7 } & & & & $f^{\prime \prime}(0)$ & $-\theta^{\prime}(0)$ & $f^{\prime \prime}(0)$ & $-\theta^{\prime}(0)$ \\
\hline 1.0 & 1.0 & 1.0 & 1.0 & 0.15559577 & 0.53157214 & 1.42056784 & 0.31161659 \\
3.0 & 1.0 & 1.0 & 1.0 & 0.17971815 & 0.53308479 & 1.56559837 & 0.39639949 \\
5.0 & 1.0 & 1.0 & 1.0 & 0.18596218 & 0.53345898 & 1.60148308 & 0.41319400 \\
1.0 & 0.0 & 1.0 & 1.0 & 0.15559577 & 0.53450902 & 1.42056784 & 0.50054887 \\
1.0 & 2.0 & 1.0 & 1.0 & 0.15559577 & 0.52863526 & 1.42056784 & 0.12268432 \\
1.0 & 4.0 & 1.0 & 1.0 & 0.15559577 & 0.52276151 & 1.42056784 & -0.25518022 \\
1.0 & 1.0 & 0.0 & 1.0 & 0.44713472 & 0.51581769 & 2.85295128 & -1.16598840 \\
1.0 & 1.0 & 1.0 & 1.0 & 0.15559577 & 0.53157214 & 1.42056784 & 0.31161659 \\
1.0 & 1.0 & 2.0 & 1.0 & 0.09387069 & 0.53275447 & 0.87830600 & 0.45792614 \\
1.0 & 1.0 & 1.0 & 0.0 & 0.15559577 & 1.14196015 & 1.42056784 & 0.62391808 \\
1.0 & 1.0 & 1.0 & 2.0 & 0.15559577 & 0.34641187 & 1.42056784 & 0.20766841 \\
1.0 & 1.0 & 1.0 & 4.0 & 0.15559577 & 0.20417385 & 1.42056784 & 0.12456459 \\
\hline
\end{tabular}

The values of skin friction coefficient and local Nusselt number for different values of the several physical parameters are presented in Table 2 . It is shown that as the Casson parameter $\beta$ increasing, the skin friction coefficient and the heat transfer are increasing for both stretching and shrinking surfaces. Moreover, the increase of the Casson parameter $\beta$ causes an increase in yield stress which it allows high resistance to the motion of fluid on the surface and consequently increase the rate of 
heat transfer. Moreover, the skin friction coefficient for Eckert number $E c$ remains constant for both surfaces as predicted since it is not in the momentum equation. However, the rate of heat transfer decreases as Eckert number $E c$ increases. The positive value indicates the heat is transferred from the surface to the Casson fluid, while the negative sign explains the opposite meaning.

The skin friction coefficient and the heat transfer rate decrease and increases respectively when the velocity slip $\omega$ is increased for both sheets. The result of the skin friction coefficient and local Nusselt number for both stretching and shrinking sheets with three different values of thermal slip parameter are also showed in Table 2. The results are consistent as $E c$ where the skin friction coefficient does not show any changes as the value of the thermal slip parameter increasing. However, the rate of skin friction shows a less drag when the sheet is stretched compared to when the sheet is shrunk. Similar conclusion is observed as $E c$ when the rate of heat transfer also decreases when the thermal slip is decreased. In a nutshell, all the pertinent parameters significantly impact on the velocity and the temperature profiles especially when the plate is shrunk. This might due to its unique backflow characteristic.

\section{Conclusions}

This research discusses the problem of radiative boundary layer flow over an exponentially permeable slippery Riga plate immersed in a Casson fluid with velocity and thermal slip effects at the boundary. The study is extended and modified from Haldar et al., [15] by added some terms to observe more into the real applications. The similarity variables are used to transform the governing equations of partial differential equations corresponding to the momentum and thermal energy equations into ordinary differential equations. The existence of the results is clearly presented and described by considering every physical parameter to determine the behavior of the velocity and temperature profiles as well as skin friction coefficient and local Nusselt number respectively. In a nutshell, all the pertinent parameters significantly impact on the velocity and the temperature profiles especially when the plate is shrunk. This might due to its unique backflow characteristic.

\section{References}

[1] Casson, N. "A flow equation for pigment-oil suspensions of the printing ink type." Rheology of disperse systems (1959).

[2] Crane, Lawrence J. "Flow past a stretching plate." Zeitschrift für angewandte Mathematik und Physik ZAMP 21, no. 4 (1970): 645-647.

https://doi.org/10.1007/BF01587695

[3] Wang, C. Y. "Liquid film on an unsteady stretching surface." Quarterly of Applied Mathematics 48, no. 4 (1990): 601-610.

https://doi.org/10.1090/qam/1079908

[4] Pramanik, S. "Casson fluid flow and heat transfer past an exponentially porous stretching surface in presence of thermal radiation." Ain Shams Engineering Journal 5, no. 1 (2014): 205-212. https://doi.org/10.1016/i.asej.2013.05.003

[5] Rawi, N. A., M. R. Ilias, Y. J. Lim, Z. M. Isa, and S. Shafie. "Unsteady mixed convection flow of Casson fluid past an inclined stretching sheet in the presence of nanoparticles." In Journal of Physics: Conference Series; IOP Publishing: Bristol, UK, vol. 890, p. 012048. 2017. https://doi.org/10.1088/1742-6596/890/1/012048

[6] Yoshimura, Ann, and Robert K. Prud'homme. "Wall slip corrections for Couette and parallel disk viscometers." Journal of Rheology 32, no. 1 (1988). https://doi.org/10.1122/1.549963

[7] Srinivas, S., P. B. A. Reddy, and B. S. R. V. Prasad. "Non-Darcian Unsteady Flow of a Micropolar Fluid over a Porous Stretching Sheet with Thermal Radiation and Chemical Reaction." Heat Transfer-Asian Research 44, no. 2 (2015): 172-187.

https://doi.org/10.1002/htj.21090 
[8] Nasir, Nor Ain Azeany Mohd, Anuar Ishak, and loan Pop. "Stagnation point flow and heat transfer past a permeable stretching/shrinking Riga plate with velocity slip and radiation effects." Journal of Zhejiang University-SCIENCE A 20, no. 4 (2019): 290-299.

https://doi.org/10.1631/jzus.A1800029

[9] Hussanan, Abid, Mohd Zuki Salleh, Ilyas Khan, and Sharidan Shafie. "Analytical solution for suction and injection flow of a viscoplastic Casson fluid past a stretching surface in the presence of viscous dissipation." Neural computing and applications 29, no. 12 (2018): 1507-1515.

https://doi.org/10.1007/s00521-016-2674-0

[10] Soid, Siti Khuzaimah, Anuar Ishak, and Ioan Pop. "Unsteady MHD flow and heat transfer over a shrinking sheet with ohmic heating." Chinese journal of physics 55, no. 4 (2017): 1626-1636.

https://doi.org/10.1016/i.cjph.2017.05.001

[11] Bhattacharyya, Krishnendu. "Dual solutions in unsteady stagnation-point flow over a shrinking sheet." Chinese Physics Letters 28, no. 8 (2011): 084702.

https://doi.org/10.1088/0256-307X/28/8/084702

[12] Requilé, Y., S. C. Hirata, and M. N. Ouarzazi. "Viscous dissipation effects on the linear stability of Rayleigh-BénardPoiseuille/Couette convection." International Journal of Heat and Mass Transfer 146 (2020): 118834.

https://doi.org/10.1016/j.ijheatmasstransfer.2019.118834

[13] Islam, Muhammad Minarul, Sheela Khatun, Md Tusher Mollah, and Md Mahmud Alam. "Fluid flow along the Riga plate with the influence of magnetic force in a rotating system." In AIP Conference Proceedings, vol. 2121, no. 1, p. 050002. AIP Publishing LLC, 2019.

https://doi.org/10.1063/1.5115889

[14] Loganathan, Parasuraman, and Krishnamurthy Deepa. "Electromagnetic and radiative Casson fluid flow over a permeable vertical Riga-plate." Journal of Theoretical and Applied Mechanics 57 (2019).

https://doi.org/10.15632/jtam-pl/112421

[15] Haldar, Syamantak, Swati Mukhopadhyay, and G. C. Layek. "Flow and heat transfer of Casson fluid over an exponentially shrinking permeable sheet in presence of exponentially moving free stream with convective boundary condition." Mechanics of Advanced Materials and Structures 26, no. 17 (2019): 1498-1504. https://doi.org/10.1080/15376494.2018.1444219

[16] Soid, Siti Khuzaimah, Anuar Ishak, and Ioan Pop. "MHD stagnation-point flow over a stretching/shrinking sheet in a micropolar fluid with a slip boundary." Sains Malaysiana 47, no. 11 (2018): 2907-2916.

https://doi.org/10.17576/jsm-2018-4711-34

[17] Mahanthesh, B., I. L. Animasaun, Mohammad Rahimi-Gorji, and Ibrahim M. Alarifi. "Quadratic convective transport of dusty Casson and dusty Carreau fluids past a stretched surface with nonlinear thermal radiation, convective condition and non-uniform heat source/sink." Physica A: Statistical Mechanics and its Applications 535 (2019): 122471.

https://doi.org/10.1016/j.physa.2019.122471

[18] Gbadeyan, J. A., E. O. Titiloye, and A. T. Adeosun. "Effect of variable thermal conductivity and viscosity on Casson nanofluid flow with convective heating and velocity slip." Heliyon 6, no. 1 (2020): e03076.

https://doi.org/10.1016/i.helivon.2019.e03076

[19] Muravleva, Larisa. "Squeeze flow of Bingham, Casson and Herschel-Bulkley fluids with yield slip at the wall by accelerated augmented Lagrangian method." Journal of Non-Newtonian Fluid Mechanics (2020): 104320. https://doi.org/10.1016/i.jnnfm.2020.104320

[20] Mukhopadhyay, Swati. "Effects of thermal radiation on Casson fluid flow and heat transfer over an unsteady stretching surface subjected to suction/blowing." Chinese Physics B 22, no. 11 (2013): 114702.

https://doi.org/10.1088/1674-1056/22/11/114702

[21] Mukhopadhyay, Swati, and Iswar Chandra Mandal. "Boundary layer flow and heat transfer of a Casson fluid past a symmetric porous wedge with surface heat flux." Chinese Physics B 23, no. 4 (2014): 044702. https://doi.org/10.1088/1674-1056/23/4/044702

[22] Brewster, M. Quinn. Thermal radiative transfer and properties. John Wiley \& Sons, 1992. 